the cervicovaginal epithelium, vaginal flora, and inflammatory markers.

\section{P2-S2.14 ALCOHOL ABUSE AND SEXUAL VICTIMISATION AMONG FEMALE SEX WORKERS IN CHINA}

doi:10.1136/sextrans-2011-050108.310

${ }^{1} Y$ Hong, ${ }^{1} \mathrm{C}$ Zhang, ${ }^{2} \mathrm{X}$ Li. ${ }^{1}$ Texas A\&M Health Science Center, College Station, USA;

${ }^{2}$ Wayne State University School of Medicine, Detroit, USA

Background Global literature suggests women often have higher sexual risks under the influence of alcohol abuse. However, data are limited from vulnerable population such as the female sex workers (FSW) in developing countries. The current study aims to fill out the literature gap by examining the association between alcohol abuse and sexual victimisation among FSWs in China.

Methods A cross-sectional survey was conducted among 1022 FSWs who were recruited through community outreach from nine different types of entertainment establishments in Guangxi, China. The FSWs completed a self-administered survey on their demographic, information, alcohol use/abuse behaviours (AUDIT), and sexual victimisation experience. Multivariate regression was employed to assess the relationship between alcohol abuse and sexual victimisation.

Results FSWs who were younger, less educated, never married, and working in an alcohol-serving establishment were more likely to have higher risks of alcohol abuse $(\mathrm{p}<0.05)$. FSWs who were at higher risks of alcohol abuse reported significantly higher sexual victimisation experience $(p<0.001)$. Multivariate models indicated significant relationships between alcohol abuse and sexual victimisation experience while controlling for potential demographic confounders, such as having been made drunk by client $(\mathrm{aOR}=1.10$, $95 \%$ CI 1.07 to 1.16$)$, "having been stripped off ( $\mathrm{aOR}=1.07,95 \% \mathrm{CI}$ $1.02,1.13)$ ", "having been taking advantages $(\mathrm{aOR}=1.08,95 \%$ $\mathrm{CI}=1.05$ to 1.12$)$ ", "having been asked for extra demands $(\mathrm{aOR}=1.08,95 \% \mathrm{CI} 1.05$ to 1.11$)$ ", and "having been sexually assaulted ( $\mathrm{aOR}=1.08,95 \%$ CI 1.04 to 1.13 )".

Conclusion Alcohol abuse was prevalent and associated with sexual victimisation experience among FSWs in China. Culturally appropriate interventions are urgently needed to reduce alcohol use related sexual risks among this vulnerable population.

\section{P2-S2.15 TYPOLOGY OF FEMALE SEX WORKERS AND ASSOCIATION WITH HIV RISKS: EVIDENCE FROM CHINA}

doi:10.1136/sextrans-2011-050108.311

${ }^{1} Y$ Hong, ${ }^{2} X$ Li. ${ }^{1}$ Texas A\&M Health Science Center, College Station, USA; ${ }^{2}$ Wayne State University School of Medicine, Detroit, USA

Background The fast growing heterosexual transmission of HIV in China has drawn global attention. Millions of female sex workers (FSW) play a critical role in the escalating epidemic. Existing studies on FSW in China have typically used venue, income or venue to categorise FSW and none developed a data-driven typology based FSW's HIV risks.

Methods A cross-sectional survey was administered among 1022 FSW recruited from 60 different types of entertainment establishments or personal service sectors in southwest China. FSW's HIV risk was measured by a composite score of 12 items on condom use, HIV testing, STD infection, drug abuse. We used multiple regression and graphics to illustrate the relationship between the
HIV risk and key FSW characteristics including age, working venue, and income.

Results Unlike previous studies that assumed a linear relationship between HIV risk and age or income, our data revealed that the relationships between HIV risk or income varied by venue. For example, older FSW in clubs or massage parlour reported lower level of HIV risks compared to their counterparts whereas older FSW in saner or KTV reported higher level of HIV risks; but age was not significantly associated with HIV risk for FSW working in hair salons or streets. Income was positively associated with HIV risks; however, the association reversed after the income reached $1500 ¥(\$ 225)$. None of the key variables such as age, income, or venue can predict HIV risks singularly; in addition, all three variables need to be integrated to devise the appropriate typology.

Conclusion Our study suggests that it was too simplistic to categorise FSW HIV risks by a single indicator; the interactions between individual and environmental factors underscored the importance of multiple indicators for typology. Interventions to reduce HIV risks in FSW in China could be more focused and targeted those at highest risks.

\section{P2-S2.16 IMPACT OF DURATION OF EXPOSURE TO SEX WORK ON HIV PREVALENCE IN 23 DISTRICTS OF SOUTHERN INDIA}

doi:10.1136/sextrans-2011-050108.312

${ }^{1} \mathrm{M}$ Pickles, ${ }^{1} \mathrm{M}$ C Boily, ${ }^{2} \mathrm{~K}$ Deering, ${ }^{3} \mathrm{P}$ Vickerman, ${ }^{4} \mathrm{~B} \mathrm{M}$ Ramesh, ${ }^{5} \mathrm{R}$ Washington, ${ }^{4} \mathrm{~S}$ Isac, ${ }^{6} \mathrm{R}$ Adhikary, ${ }^{7} \mathrm{R}$ S Paranjape, ${ }^{8} \mathrm{M}$ Alary. ${ }^{1}$ Imperial College, London, UK; ${ }^{2}$ University of British Columbia, Canada; ${ }^{3}$ LSHTM, UK; ${ }^{4}$ Karnataka Health Promotion Trust, Bangalore, India; ${ }^{5}$ St John's Research Institute, Bangalore, India; ${ }^{6}$ Family Health International, India; ${ }^{7}$ National AIDS Research Institute, Pune, India; ${ }^{8}$ Centre hospitalier affilié universitaire de Québec, Quebec, Canada

Background HIV prevalence among female sex workers (FSW) in various districts of Andhra Pradesh (AP), Karnataka (KA), Maharashtra $(\mathrm{MH})$, Tamil Nadu (TN) ranged between 8 and $26 \%, 10$ and $34 \%, 6$ and $38 \%, 2$ and $13 \%$, respectively, in $2005-2006$. The duration of selling or buying sex has been suggested as a key determinant that may explain ecological difference in HIV prevalence. Thus, we estimated FSW duration across geographically distinct districts and assessed its impact on HIV transmission.

Methods Cross-sectional FSW survey data from 23 districts of $\mathrm{MH}$, TN, KA, AP (2005-2006) (IBBA), collected as part of the evaluation of Avahan, the India AIDS initiative, were used to estimate FSW duration (crude duration=difference between age at survey and age of entry into sex work). "Corrected" durations that adjust for the censored nature of the data (as FSW are still in sex work) were estimated for FSW using a birth-and-death dynamical model (with 9 duration compartments) fitted by maximum likelihood to each district-specific FSW IBBA duration distribution. A deterministic model of HIV transmission among FSW/clients was parameterized/ fitted to Belgaum IBBA data with crude FSW duration of 9.6 years for FSW. FSW duration was thereafter varied between 1 and 33 years to assess its influence on model HIV prevalence, for different assumed client duration (4-17 years). Each individual leaving was replaced by a new susceptible person.

Results Mean crude FSW durations ranged between 3.7 and 9.6 (median 3-7) years across districts compared to 4.3 (Bangalore, Thane street-based) and 12.5 (Belgaum) after correction. District average corrected durations tended to be shorter for TN (6 years) and longer for $\mathrm{MH}$ (9 years). Within-district differences (ratios) between corrected and crude durations ranged between 0.5 and 3 years (1.1-1.3-fold). Observed differences in FSW duration across 